\title{
Characterization of influenza A(H1N1)pdm09 viruses in Germany in season 2019-2020 - co-circulation of an antigenic drift variant
}

\author{
Marianne Wedde ${ }^{1}$, Djin-Ye Oh${ }^{1}$, Silke Buda ${ }^{2}$, Andrea Thürmer ${ }^{1}$, Sandra Kaiser ${ }^{1}$, Barbara \\ Biere $^{3}$, Susanne Duwe ${ }^{1}$, Janine Reiche ${ }^{1}$, Thorsten Wolff ${ }^{1}$, and Ralf Dürrwald ${ }^{1}$ \\ ${ }^{1}$ Robert Koch Institut \\ ${ }^{2}$ Robert Koch-Institute \\ ${ }^{3}$ Robert Koch Inst
}

August 16, 2021

\begin{abstract}
Background Influenza A(H1N1)pdm09 virus entered the human population in 2009 and evolved within this population for more than ten years. Despite genetic evolution no remarkable changes in the antigenic reactive pattern of these viruses were observed so far. Methods Primary respiratory samples of the German influenza virological sentinel were investigated by qPCR. Influenza virus-positive samples were characterized genetically and antigenetically. Results In December 2019, a antigenic drift variant characterized by an N156K substitution in the hemagglutinin of influenza A(H1N1)pdm09 virus emerged in Germany, which exhibited a reactivity to ferret antiserum that was an average $6 \log 2$ lower than the vaccine virus A/Brisbane/02/2018 and the other $\mathrm{A}(\mathrm{H} 1 \mathrm{~N} 1)$ pdm09 viruses circulating in the influenza season 2019-2020. These viruses accounted for $20 \%$ of all $\mathrm{A}(\mathrm{H} 1 \mathrm{~N} 1) \mathrm{pdm} 09$ viruses characterized in the German influenza sentinel. Patients infected with these viruses had a shorter median time period of medical consultation after onset of symptoms and were more frequently treated with neuraminidase inhibitors in comparison to patients infected with other A(H1N1)pdm09 viruses. Conclusions This parallel circulation of two antigenic variants of $\mathrm{A}(\mathrm{H} 1 \mathrm{~N} 1)$ pdm09 viruses which differ remarkably in their antigenic reactive pattern contributes to a greater variability in circulating influenza viruses and challenges vaccination.
\end{abstract}

\section{INTRODUCTION}

In 2009 a new H1N1 influenza A virus emerged in Mexico and spread quickly through the global human population, thereby causing the first influenza pandemic of the $21^{\text {st }}$ century and the seasonal $\mathrm{A}(\mathrm{H} 1 \mathrm{~N} 1)$ viruses circulating previously have since been replaced by this pandemic A(H1N1)pdm09 strain (1-3). With respect to their antigenic properties, A(H1N1)pdm09 viruses have remained closely related to the first viruses that emerged in 2009 ; the initial vaccine virus A/California/7/2009 was replaced only twice: in season 2017-2018 by Michigan/45/2015 and in season 2019-2020 by Brisbane/02/2018 (https://www.who.int/influenza/vaccines/virus/recommendations/en/ ). A high level of antigenic reactivity remained between circulating viruses and antisera to the initial vaccine virus of 2009 (https://www.crick.ac.uk/sites/default/files/2018-07/crick_nh_vcm_report_feb_2017_v2.pdf). Accordingly, the hemagglutinin (HA) of A(H1N1)pdm09 viruses is similar to that of 1918 pandemic influenza A(H1N1) viruses (4): While it lacks the multiple N-glycosylation sites that were associated with antigenic drift of the previous seasonal $\mathrm{A}(\mathrm{H} 1 \mathrm{~N} 1)$ viruses, it retains codon-motifs that are able to become N-glycosylated (5). Therefore it is deemed probable that antigenic drift of $\mathrm{A}(\mathrm{H} 1 \mathrm{~N} 1)$ pdm09 viruses will occur in the near future (5). A(H1N1)pdm09 viruses are strong antigens in humans and therefore vaccine effectiveness achieved by the $\mathrm{A}(\mathrm{H} 1 \mathrm{~N} 1) \mathrm{pdm} 09$ viruses is superior to that observed for $\mathrm{A}(\mathrm{H} 3 \mathrm{~N} 2)$ influenza viruses $(6,7)$. Here, we report the emergence of antigenic drift variants that may be the first signs of the predicted antigenic evolution of $\mathrm{A}(\mathrm{H} 1 \mathrm{~N} 1) \mathrm{pdm} 09$ viruses. Antigenic drift may have substantial clinical and therapeutic implications, 
as has been exemplified by the drift of the previous $\mathrm{A}(\mathrm{H} 1 \mathrm{~N} 1)$ seasonal viruses, which led to neuraminidase inhibitor resistance during the last years of their circulation in the human population $(8,9)$. Thus, continued surveillance of $\mathrm{A}(\mathrm{H} 1 \mathrm{~N} 1)$ pdm09 viruses is of major importance.

\section{MATERIAL AND METHODS}

\section{Ethics Statement}

All investigations were approved by Charité Universitätsmedizin Berlin Ethical Board (reference EA2/126/11 and EA2/218/19). Sentinel surveillance is covered by German legislation (§13, §14, Protection against Infection Act). Consent was obtained from patients or their parents or guardians.

\section{Samples}

Primary respiratory samples were collected between October week 40/2019 and March week 14/2020 in the context of the Arbeitsgemeinschaft Influenza (AGI) virological sentinel, including nasal or throat swabs from a total of 5080 patients presenting with acute respiratory illness. Of these, 413 were A(H1N1)pdm09 positive. In addition, 471 influenza positive samples (isolates) were provided by diagnostic laboratories distributed all over Germany. Of these, 218 were A(H1N1)pdm09 positive (Supplemental Table 1).

\section{qPCR}

RNA was extracted from $200 \mu \mathrm{L}$ sample material (homogenized, if applicable) using the MagNA Pure 96 (or 24) DNA and Viral NA Small Volume Kit or MagNA Pure 24 Total Nucleic Acid Kit (Roche) and eluted in $50 \mu \mathrm{L}$ elution buffer. $25 \mu \mathrm{L}$ of extracted RNA were subjected to cDNA synthesis in a total reaction volume of $40 \mu \mathrm{L}$, applying random hexamer primers and 200U M-MLV Reverse Transcriptase (Invitrogen). Synthesised cDNA was diluted $1: 1$ with $\mathrm{H}_{2} \mathrm{O}$ to a total volume of $80 \mu \mathrm{L}$ to allow robotic pipetting of 384-well PCR plates.

qPCR was carried out on LC480II real-time PCR thermal cyclers (Roche) in a total reaction volume of 15 $\mu \mathrm{L}$. The reaction contained $1 \mathrm{x}$ PCR buffer, $4 \mathrm{mmol} / \mathrm{L} \mathrm{MgCl}_{2}, 1 \mathrm{mmol} / \mathrm{L}$ dNTP (ThermoFisher) with dUTP (GE Healthcare), 600ng BSA (ThermoFisher), 0.3-1U Platinum Taq Polymerase (ThermoFisher), variable concentrations of primers and probes (available on request), and $5 \mu \mathrm{L}$ of the prediluted cDNA. After 5 minutes at $95^{\circ} \mathrm{C}$ for Taq DNA polymerase activation, a total of 45 cycles consisting of denaturation at $95^{\circ} \mathrm{C}$ for 15 seconds and annealing at $60^{\circ} \mathrm{C}$ for 30 seconds were performed. After the run, data were analysed using the LightCycler software version 1.5.1.62.

\section{Sequencing}

Viral RNA was extracted using the ReliaPrep Cell Mini Kit (Promega) using a protocol adapted for extraction of RNA from seasonal influenza viruses (using qPCR positive specimens. First, $200 \mu$ l diluted specimen, $50 \mu \mathrm{l}$ water and $250 \mu \mathrm{l}$ freshly prepared buffer (BL+TG lysis buffer) were mixed by vortexing. Next, $85 \mu \mathrm{l}$ of isopropanol $(100 \%$, Roth) were added and mixed by vortexing. All subsequent steps (including DNase digestion) were performed according to manufacturer's instructions. Finally, the RNA was eluted in $60 \mu \mathrm{l}$ of water. Next, multisegment reverse transcription-PCR was performed according to Zhou et al. (10), using viral $14 \mu \mathrm{l}$ RNA and SuperScript III One-Step RT-PCR System with Platinum Taq High Fidelity DNA Polymerase (Invitrogen).

One-Step RT-PCR products were purified using SPRIselect beads (Beckman Coulter) according to the manufacturer's instructions, with a ratio of 0.6. Then, DNA quantification was performed using Qubit dsDNA kit and a Qubit Fluorometer (ThermoFisher).

NGS was performed using 1 ng purified One-Step RT-PCR product, Nextera XT DNA Library Preparation Kit and subsequently the ISeq platform (Illumina). Trimming, reference mapping and generation of consensus sequences (threshold 90\%) were done with Geneious software (11.1.5) and influenza A(H1N1)pdm09 genome sequences (minority variants [?]10\%) were deposited in GISAID (www.GISAID.org).

\section{Phylogenetic analysis}


Phylogenetic evaluation of HA and NA genes was done using Mega 7, neighbour-joining method, phylogeny test by bootstrap method and 1000 replications, Kimura 2 substitution model and partial deletion (site coverage cutoff $5 \%$ ). The viruses were compared with $\mathrm{A}(\mathrm{H} 1 \mathrm{~N} 1) \mathrm{pdm} 09$ reference viruses according to McCauley et al. (2019) (11). The identified substitutions (see FluSurver analysis) were assigned to protein domains (region of antigenic sites and receptor binding sites) according to the FluSurver database and Igarashi et al. and Yang et al. $(5,12)$.

\section{FluSurver analysis}

For further analysis of the HA and NA genes the https://flusurver.bii.a-star.edu.sg/ database was used (i) to detect substitutions relative to the outgroup in the phylogenetic analysis (A/Michigan/45/2015), (ii) to display the position of the mutation(s) in structural models and highlight if mutations are close to common drug, host receptor or antibody binding sites; or if a glycosylation motif is lost or created through a mutation relative to the closest reference A/Brisbane/02/2018.

\section{Virus isolation}

Virus isolation was carried out on MDCK-SIAT cells (13).

\section{Antigenic analysis}

The viruses were investigated for their antigenic properties by hemagglutination inhibition (HI) assay with ferret antisera against the vaccine strains of the influenza season 2019-2020 and A(H1N1)pdm09 vaccine viruses of the influenza season 2020-2021. HI assays employed turkey and guinea pig red blood cells (the latter for H3N2); viruses were adjusted to 8 hemagglutinating units for analysis. In order to generate ferret antisera, intranasal infection of ferrets was performed with $10^{5} \mathrm{TCID}_{50}$ of each of the vaccine viruses and blood was sampled 14 days after infection.

\section{Results}

Over the course of the 2019-2020 influenza season, an increasing number of influenza A(H1N1)pdm09 viruses exhibited decreased reactivity towards ferret antiserum against the vaccine virus A/Brisbane/02/2018 (Figure 1A), indicating an antigenic profile distinct from the vaccine virus. For these viruses, an average $6 \log _{2}$ reduction in reactivity compared to the vaccine virus was observed (Figure $1 \mathrm{~A}$ ). These viruses emerged in December 2019 and continued to circulate until March 2020, when influenza activity in Germany declined due to the lockdown and safety measures in response to the SARS-CoV-2 pandemic (Figure 2). In a total, of $631 \mathrm{~A}(\mathrm{H} 1 \mathrm{~N} 1)$ pdm09 viruses detected in 2019-2020 the antigenic profiles of 417 viruses were determined and $\mathrm{HI}$ assay results indicated good vaccine coverage for influenza viruses of other subtypes $(\mathrm{A}(\mathrm{H} 3 \mathrm{~N} 2)$ and B) circulating in Germany (Figure 1B-D).

164 A(H1N1)pdm09 influenza viruses (mainly whole genomes) were sequenced by NGS in 2019-2020. The HA gene analysis of $\mathrm{A}(\mathrm{H} 1 \mathrm{~N} 1)$ pdm09 viruses circulating between week 42/2019 and 5/2020 revealed that the majority of viruses ( 69 out of $71=97 \%$ ) belonged to the 6B.1A5A clade, represented by A/Norway/3433/2018 and characterized by the clade-specific substitutions N129D and T185I in HA1. The remaining two viruses fell into the 6B1.A7 clade, displaying the K302T substitution in HA1 and the I77M, N169S, E179D substitutions in HA2. Among the 6B1.A5A-viruses, five variants were identified, which carried additional substitutions, namely D187A, Q189E in HA1 (51\%, cluster 1); R205K in HA1 (3\%, cluster 2); N156K, A195E in HA1 (7\%, cluster 3); K130N, N156K, L161I, V250A in HA1, E179D in HA2 and L8M in the signal peptide partially with K209M substitution in HA1 (20\%, cluster 4); and V193A in HA2 (15\%, cluster 5; Figure 3A, Table 1 ). HA gene analysis demonstrated that all viruses with reduced reactivity towards antiserum against the vaccine virus A/Brisbane/02/2018 carried the N156K substitution, which is located in the Sa antigenic site on the hemagglutinin. Interestingly, the N156K substitution was detected in two different virus clusters (3 and 4) and also in one virus of cluster 1 (A/MVP/2/2020). Although these cluster 1,3 and 4 viruses had a different genetic background, all N156K-viruses showed reduced reactivity in the hemagglutination inhibition test (Figure 1A). For a 6B.1A5A virus (A/THR/125/2019, near cluster 4) with polymorphisms at 
amino acid position $156(\mathrm{~N} 156 \mathrm{~N} / \mathrm{K})$ and at other positions (L8L/M in signal peptide, K130K/N, V250V/A in HA1, E179E/D, V193V/A in HA2), an only slight HI-titer reduction was observed ([?]4-fold, Figure 3A).

All test viruses for which the genetic group or subgroup were known, with collection

dates after 2018-08-31, fell into clade 6B.1 which is defined by the amino acid

substitutions S84N, S162N (introducing a potential N-linked glycosylation site) and

I216T in HA1 with the viruses clustering into a genetic subclade designated as 6B.1A

and defined by the HA1 amino acid substitutions S74R ,S164T (which alters the

glycosylation motif at residues 162 to 164 ) and I295V .

The NA gene analysis showed a phylogeny similar to that based on the HA gene (Fig. 3B). The majority of viruses clustered with 6B.1A5A-viruses (97\%), carrying the Q51K, T74S, I389K, T452I substitutions. The two 6B.1A7-viruses (3\%) displayed the M314I and F322L substitutions in NA. Consistent with HA gene phylogeny, five clusters could be discerned: Cluster 1 (S52N, partially also I40T, T72N, 49\%), cluster 3 (I396M, 4\%) and cluster 4 (M19T, Y66F, N222K, partially also T72I, 23\%) exhibited cluster-specific substitutions, whereas cluster $2(3 \%)$ and $5(17 \%)$ did not. Of interest, two viruses clustered differently in the NA gene than in the HA gene: A/BWB/11/2020 (HA cluster 1) clustered with viruses of cluster 4 in the NA gene and A/SAS/3/2020 (HA cluster 3) did not belong to any of the five cluster in the NA gene (Figure 3B).

HA and NA sequences were examined using the https://flusurver.bii.a-star.edu.sg/ online tool to assess for substitutions which are located close to common drug, host receptor or antibody binding sites; or which promote the loss or creation of a glycosylation motif (relative to the closest reference A/Brisbane/02/2018). One virus (A/NRW/142/2019) featured the S247N substitution, which is described to be associated with mild drug resistance (14). This virus belonged to the HA-N156K variant (cluster 4) and displayed HI titers that were $>4$ fold reduced. Three viruses carrying the HA-N156K substitution, belonging to clusters 1 (A/MVP/2/2020), 3 (A/MVP/12/2019) and 4 (A/NRW/142/2019), were further analyzed, using flusurver to deduce the localization of the identified substitutions in a structural model, as shown in Figure 4A-F, which indicates substitutions relative to the closest reference A/Brisbane/02/2018.

Next, cross neutralization was assessed via HI assay, including specifically generated ferret antisera against A(H1N1)pdm09 HA-N156K . These experiments revealed clear differences between A(H1N1)pdm09 HAN156K and other $\mathrm{A}(\mathrm{H} 1 \mathrm{~N} 1)$ pdm09 viruses (Table 2). Although viruses of both groups are still recognized by antisera against either group, antigenic reactivity between these variants is reduced 3-7 $\log _{2}$ fold.

To gain first insight into a potential clinical impact of the HA-N156K mutation, patient data were evaluated, assessing specifically whether (i) these viruses were preferentially detected in certain patient groups (age group, patients with chronic disease, patients with pneumonia, vaccinated patients) and / or (ii) patients were prescribed antiviral agents (as a proxy of disease severity) and / or (iii) time to physician consultation after symptoms onset was short (as another proxy of disease severity) (Supplemental Table 2). Patients infected with viruses carrying the HA-H156K mutation were more frequently treated with neuraminidase inhibitors than those infected with other A(H1N1)pdm09 viruses. Moreover, the time delay between symptom onset and physician consultation that was observed for these patients was shorter. No other differences were noted between patients infected with A(H1N1)pdm09 HA-N156K viruses and those infected with other A(H1N1)pdm09 viruses.

\section{Discussion}

The hemagglutinin molecule of H1N1 influenza possesses several important antigenic sites, which are targeted by hemagglutination inhibiting (HI) antibodies $(15,16)$. These epitopes are named Sa, Sb, Ca1, Ca2, and $\mathrm{Cb}(4)$. They are subjected to antibody-mediated immune pressure and are therefore variable (16). Strain(S)-specific sites are located at the top of the hemagglutinin head and display high levels of variation, 
whereas cross-reactive(C)-sites display less variability, most likely due to their more sideward (less exposed) location on the hemagglutinin. The antigenic sites are targeted by different species in a different manner, a phenomenon called immunodominance (17). HA-reactive antibodies of humans are preferentially affected by both sites $\mathrm{Sb}$ and $\mathrm{Sa}$, whereas those of ferrets are mainly affected by site Sa (17). Therefore, similarities exist between both species regarding epitope Sa. HA antigenic drift occurs in response to increasing population immunity induced by infection or vaccination, which acts as a strong evolutionary pressure (18). The influenza season 2019-2020 has been the third consecutive season featuring prominent circulation of A(H1N1)pdm09 viruses in Germany: In 2017-2018, shortly after B/Yamagata influenza viruses had passed their peak, A(H1N1)pdm09 influenza viruses surged, effectively constituting a second influenza wave within that season. In 2018-2019 A(H1N1)pdm09 viruses circulated in parallel with $\mathrm{A}(\mathrm{H} 3 \mathrm{~N} 2)$ viruses. In 20192020, a similar pattern of $\mathrm{A}(\mathrm{H} 1 \mathrm{~N} 1) \mathrm{pdm} 09$ and $\mathrm{A}(\mathrm{H} 3 \mathrm{~N} 2)$ viruses circulating in parallel was observed, which was followed by the circulation of B/ Victoria influenza viruses. Circulation of A(H1N1)pdm09 viruses over three successive seasons thus far may have induced higher-than-usual population immunity levels, thus augmenting evolutionary pressure on $\mathrm{A}(\mathrm{H} 1 \mathrm{~N} 1)$ pdm09 viruses. The emergence of antigenic variants displaying substantially reduced reactivity towards antisera against vaccine viruses would be a logical consequence of this pressure. The data reveal a clear intra-seasonal antigenic drift within the influenza A(H1N1)pdm09 subtype, which is associated with the N156K substitution in the Sa antigenic site of the hemagglutinin. Importantly, this kind of antigenic drift has been predicted by Guarnaccia et al. (2013), based on the results of sequential passaging studies in ferrets: HA-N156K emerged during serial passaging of A(H1N1)pdm09 virus in suboptimally vaccinated ferrets, where the N156 HA variant outgrew wildtype virus and showed altered receptor binding preferences (18). Igarashi et al. (2010) predicted similar evolutionary trends, based onin silico analyses of the previous seasonal, and historical $\mathrm{A}(\mathrm{H} 1 \mathrm{~N} 1)$ viruses (5).

A(H1N1)pdm09 viruses have thus far been highly immunogenic and in most years, vaccine effectiveness against $\mathrm{A}(\mathrm{H} 1 \mathrm{~N} 1)$ pdm09 viruses exceeded that observed for $\mathrm{A}(\mathrm{H} 3 \mathrm{~N} 2)$ and influenza $\mathrm{B}$ viruses (7). Patient data analysis did not indicate a reduction of vaccine effectiveness, probably for the following reasons: (i) there is still cross reactivity between these viruses and the vaccine virus at lower level; ii) there is probably still a high level of population immunity camouflaging effects of cross-reactivity at lower level. However, time to medical consultation medians differed by 1 day between N156K and non-N156K viruses. Although N156K is not located in the receptor binding site, it is positioned at the interface of three antibody binding sites (19-21). It has been postulated to increase receptor binding avidity, thereby supporting extensive replication (18). The shorter time to medical consultation observed in patients infected with H156K HA viruses may be an indicator of this presumed extensive replication pattern. The proportion of patients receiving antiviral therapy was higher among those infected with HA-N156K viruses, which could be related to more severe clinical courses of infection with these variants.

To date, A(H1N1)pdm09 viruses are sensitive to neuraminidase inhibitors (https://influenza.rki.de/Wochenberichte.aspx). Among the N156K viruses, only one displayed a mutation associated with mild drug resistance, similar to that observed in Australia (14).

The investigations reflect influenza $\mathrm{A}(\mathrm{H} 1 \mathrm{N1}$ )pdm09 virus hemagglutinin antigenic drift, following ten years of circulation of these viruses in the human population. These drift variants, characterized by an asparagine to lysine exchange at position 156 of the HA gene, emerged in different genetic clades during the same season, indicating convergent evolution. This HA-N156K substitution arose under conditions favoring increased immune selection pressure on $\mathrm{A}(\mathrm{H} 1 \mathrm{~N} 1) \mathrm{pdm} 09$ viruses, given three consecutive years of prominent circulation, which likely augmented population immunity. HA-N156K viruses still react with antiserum directed against the vaccine virus $\mathrm{A} /$ Brisbane/02/2018 but at substantially reduced levels; ultimately, this may result in a shorter duration of vaccine-induced immunity, which could considerably impact vaccine effectiveness and have important public health implications. These findings were taken into consideration when the decision was made on the 2020 vaccine composition: A(H1N1)pdm09 HA-N156K viruses were included in the 2020 influenza vaccines recommended for the Southern hemisphere and in 2020/21 influenza vaccines for the Northern hemisphere. In Germany, as well as in other countries, the 2019-2020 influenza season was cut short by COVID-19 targeted non-pharmaceutical interventions (NPIs) (22). Similarly, 2020/21 influenza 
activity was historically low. Thus, it remains to be seen whether these NPIs have led to the extinction of this first A(H1N1)pdm09 HA antigenic drift variant as an unintended benefit. Taken together with animal model data predicting this same amino acid exchange as the basis for immune escape (18), our findings indicate that even if HA-N156K variants have been extinguished for now, they may reemerge in future influenza seasons. This highlights the major importance of laboratory-based virological surveillance in order to monitor viral evolution, which gives rise to novel pathogen variants, thereby carrying important public health implications.

\section{Acknowledgements}

We thank Prof. Dr. Timm Harder and Prof. Dr. Martin Beer, Friedrich-Loeffler-Institute, Greifswald - Isle of Riems, Germany for establishment of ferret antisera; Mareen Adam, Susi Hafemann, Ute HopfGuevara, Carmen Karstädt-Schulze, Katja-Irena Madaj, Jeanette Milde, Bettina Mischke, Christine Spingies, Anneliese Schindel, Maria Smallfield and Nathalie Tollard for excellent technical assistance; Heike Fischer and Kathrin Seidel for outstanding sequencing support.

We thank Dr. John McCauley and Áine Rattigen, WHO Collaborating Centre, Francis Crick Institute, London for providing vaccine viruses and antisera.

\section{Conflict interests}

The authors declare that they have no conflict interests.

\section{References}

1. Mena I, Nelson MI, Quezada-Monroy F, Dutta J, Cortes-Fernandez R, Lara-Puente JH, et al. Origins of the 2009 H1N1 influenza pandemic in swine in Mexico. Elife. 2016;5.

2. Novel Swine-Origin Influenza AVIT, Dawood FS, Jain S, Finelli L, Shaw MW, Lindstrom S, et al. Emergence of a novel swine-origin influenza A (H1N1) virus in humans. N Engl J Med. 2009;360(25):2605-15.

3. Dawood FS, Iuliano AD, Reed C, Meltzer MI, Shay DK, Cheng PY, et al. Estimated global mortality associated with the first 12 months of 2009 pandemic influenza A H1N1 virus circulation: a modelling study. Lancet Infect Dis. 2012;12(9):687-95.

4. Zhang W, Qi J, Shi Y, Li Q, Gao F, Sun Y, et al. Crystal structure of the swine-origin A (H1N1)-2009 influenza A virus hemagglutinin (HA) reveals similar antigenicity to that of the 1918 pandemic virus. Protein Cell. 2010;1(5):459-67.

5. Igarashi M, Ito K, Yoshida R, Tomabechi D, Kida H, Takada A. Predicting the antigenic structure of the pandemic (H1N1) 2009 influenza virus hemagglutinin. PLoS One. 2010;5(1):e8553.

6. Lansbury LE, Smith S, Beyer W, Karamehic E, Pasic-Juhas E, Sikira H, et al. Effectiveness of 2009 pandemic influenza A(H1N1) vaccines: A systematic review and meta-analysis. Vaccine. 2017;35(16):19962006 .

7. Kissling E, Pozo F, Buda S, Vilcu AM, Rizzo C, Gherasim A, et al. Effectiveness of influenza vaccine against influenza $\mathrm{A}$ in Europe in seasons of different $\mathrm{A}(\mathrm{H} 1 \mathrm{~N} 1)$ pdm09 and the same $\mathrm{A}(\mathrm{H} 3 \mathrm{~N} 2)$ vaccine components (2016-17 and 2017-18). Vaccine X. 2019;3:100042.

8. Lackenby A, Hungnes O, Dudman SG, Meijer A, Paget WJ, Hay AJ, et al. Emergence of resistance to oseltamivir among influenza A(H1N1) viruses in Europe. Euro Surveill. 2008;13(5).

9. Meijer A, Lackenby A, Hungnes O, Lina B, van-der-Werf S, Schweiger B, et al. Oseltamivir-resistant influenza virus A (H1N1), Europe, 2007-08 season. Emerg Infect Dis. 2009;15(4):552-60.

10. Zhou B, Donnelly ME, Scholes DT, St George K, Hatta M, Kawaoka Y, et al. Single-reaction genomic amplification accelerates sequencing and vaccine production for classical and Swine origin human influenza a viruses. J Virol. 2009;83(19):10309-13. 
11. McCauley J, Rodney D, Lin YP, Xiang Z, Whittaker L, Halai C, et al. Report prepared for the WHO annual consultation on the composition of influenza vaccine for the Northern hemisphere 2019-2020. London; 20192019.

12. Yang H, Carney P, Stevens J. Structure and Receptor binding properties of a pandemic H1N1 virus hemagglutinin. PLoS Curr. 2010;2:RRN1152.

13. Matrosovich M, Matrosovich T, Carr J, Roberts NA, Klenk HD. Overexpression of the alpha-2,6sialyltransferase in MDCK cells increases influenza virus sensitivity to neuraminidase inhibitors. J Virol. 2003;77(15):8418-25.

14. Hurt AC, Lee RT, Leang SK, Cui L, Deng YM, Phuah SP, et al. Increased detection in Australia and Singapore of a novel influenza A(H1N1)2009 variant with reduced oseltamivir and zanamivir sensitivity due to a S247N neuraminidase mutation. Euro Surveill. 2011;16(23).

15. Caton AJ, Brownlee GG, Yewdell JW, Gerhard W. The antigenic structure of the influenza virus A/PR/8/34 hemagglutinin (H1 subtype). Cell. 1982;31(2 Pt 1):417-27.

16. Brownlee GG, Fodor E. The predicted antigenicity of the haemagglutinin of the 1918 Spanish influenza pandemic suggests an avian origin. Philos Trans R Soc Lond B Biol Sci. 2001;356(1416):1871-6.

17. Liu STH, Behzadi MA, Sun W, Freyn AW, Liu WC, Broecker F, et al. Antigenic sites in influenza H1 hemagglutinin display species-specific immunodominance. J Clin Invest. 2018;128(11):4992-6.

18. Guarnaccia T, Carolan LA, Maurer-Stroh S, Lee RT, Job E, Reading PC, et al. Antigenic drift of the pandemic 2009 A(H1N1) influenza virus in A ferret model. PLoS Pathog. 2013;9(5):e1003354.

19. Fleury D, Wharton SA, Skehel JJ, Knossow M, Bizebard T. Antigen distortion allows influenza virus to escape neutralization. Nat Struct Biol. 1998;5(2):119-23.

20. Barbey-Martin C, Gigant B, Bizebard T, Calder LJ, Wharton SA, Skehel JJ, et al. An antibody that prevents the hemagglutinin low pH fusogenic transition. Virology. 2002;294(1):70-4.

21. Xu R, Ekiert DC, Krause JC, Hai R, Crowe JE, Jr., Wilson IA. Structural basis of preexisting immunity to the 2009 H1N1 pandemic influenza virus. Science. 2010;328(5976):357-60.

22. Oh D-Y, Buda S, Biere B, Reiche J, Schlosser F, Duwe S, et al. Trends in respiratory virus circulation following COVID-19-targeted nonpharmaceutical interventions in Germany, January - September 2020: analysis of national surveillance data. Lancet Reg Health Eur. 2021;6:100112. doi: 10.1016/j.lanepe.2021.100112. PubMed PMID: 34124707; PubMed Central PMCID: PMCPMC8183189.

\section{Tables and Figures}

Table 1: HA gene analysis of AH1N1)pdm09 viruses. The proportion of novel HA variants in season 20192020 expressing cluster specific substitutions in HA1 and HA2 is indicated. Additionally, the proportion of viruses with reduced reactivity ( $>4$-fold versus [?]4-fold) in the hemagglutination inhibition test is shown.

\begin{tabular}{|c|c|c|c|c|c|c|c|c|}
\hline $\begin{array}{l}\text { HA gene } \\
\text { analysis }^{1} \\
\mathrm{cw}^{2} \\
42 / 2019- \\
5 / 2020\end{array}$ & Total & 6B.1A5A & $\begin{array}{l}\text { 6B.1A5A } \\
\text { Cluster } 1\end{array}$ & $\begin{array}{l}\text { 6B.1A5A } \\
\text { Cluster } 2\end{array}$ & $\begin{array}{l}\text { 6B.1A5A } \\
\text { Cluster } 3\end{array}$ & $\begin{array}{l}\text { 6B.1A5A } \\
\text { Cluster } 4\end{array}$ & $\begin{array}{l}\text { 6B.1A5A } \\
\text { Cluster } 5\end{array}$ & 6B.1A \\
\hline $\mathrm{HA1}^{3}$ & & $\begin{array}{l}\text { N129D, } \\
\text { T185I }\end{array}$ & $\begin{array}{l}+\mathrm{D} 187 \mathrm{~A}, \\
\text { Q189E }\end{array}$ & $+\mathrm{R} 205 \mathrm{~K}$ & $\begin{array}{l}+\mathbf{N 1 5 6 K} \\
\mathrm{A} 195 \mathrm{E}\end{array}$ & $\begin{array}{l}+\mathrm{K} 130 \mathrm{~N}, \\
\text { N156K, } \\
\text { L161I, } \\
\text { V250A, } \\
\text { partly } \\
\text { K209M }\end{array}$ & & K302T \\
\hline
\end{tabular}


HA gene

analysis $^{1}$

$\mathrm{cw}^{2}$

$42 / 2019$ -

$5 / 2020$

Total

6B.1A5A

6B.1A5A

6B.1A5A

6B.1A5A

6B.1A5A

HA2 $^{3}$

Cluster 1

Cluster 2 Cluster 3

Cluster 4

Cluster 5

6B.1A

+ E179D +V193A

I77M,

N169S,

All

100

1

51

3

7

20

15

E179D

viruses

(\%)

All

71

1

36

2

5

14

11

3

viruses

(n)

Viruses $\quad 51$

with

1

35

2

0

0

11

2

HI-

titer

[?]4-

fold

(n)

Viruses 18

$18 \quad 0$

$\begin{array}{ll}0 & 1^{4}\end{array}$

$1^{4} \quad 0$

5

12

0

0

with

HI-

titer

$>4$-fold

(n)

No HI- 2

titer

(n)

${ }^{1}$ RNA extracted from patient specimen. ${ }^{2}$ Calender week. ${ }^{3}$ Clade/cluster specific substitutions in HA1 and HA2. ${ }^{4} \mathrm{~A}(\mathrm{H} 1 \mathrm{~N} 1)$ pdm09 virus in cluster 1 with virus specific N156K substitution.

Table 2: Cross neutralization data (HI) of current and past $\mathrm{A}(\mathrm{H} 1 \mathrm{~N} 1)$ pdm09 influenza vaccine viruses and selected $\mathrm{A}(\mathrm{H} 1 \mathrm{~N} 1) \mathrm{pdm} 09$ viruses of the German sentinel reflect antigenic variation between $\mathrm{A}(\mathrm{H} 1 \mathrm{~N} 1) \mathrm{pdm} 09$ N156K and other $\mathrm{A}(\mathrm{H} 1 \mathrm{~N} 1) \mathrm{pdm} 09$ viruses

\begin{tabular}{llll}
\hline & $\begin{array}{l}\text { Anti A/Guandgong- } \\
\text { Maonan/SWL1536/2019 } \\
\text { A(H1N1)pdm09 }\end{array}$ & $\begin{array}{l}\text { Anti } \\
\text { A/Brisbane/2/2018 } \\
\mathrm{A}(\mathrm{H} 1 \mathrm{~N} 1 \mathrm{pdm} 09\end{array}$ & $\begin{array}{l}\text { Anti } \\
\mathrm{A} / \text { Victoria/2570/2019 } \\
\mathrm{A}(\mathrm{H} 1 \mathrm{~N} 1) \mathrm{pdm} 09 \text { N156K }\end{array}$ \\
\hline $\begin{array}{l}\text { Immune sera Viruses } \\
\text { A/Guandgong- }\end{array}$ & 2560 & 5120 & 640 \\
$\begin{array}{l}\text { Maonan/SWL1536/2019 } \\
\text { A(H1N1)pdm09 }\end{array}$ & & & \\
$\begin{array}{l}\text { A/Brisbane/2/2018 } \\
\text { A(H1N1)pdm09 }\end{array}$ & 1280 & 2560 & 20 \\
$\begin{array}{l}\text { A/Baden- } \\
\text { Württemberg/139/20 }\end{array}$ & 640 & 2560 & 20 \\
A(H1N1)pdm09 & & &
\end{tabular}




\begin{tabular}{|c|c|c|c|}
\hline Immune sera Viruses & $\begin{array}{l}\text { Anti A/Guandgong- } \\
\text { Maonan/SWL1536/2019 } \\
\text { A(H1N1)pdm09 }\end{array}$ & $\begin{array}{l}\text { Anti } \\
\text { A/Brisbane/2/2018 } \\
\text { A(H1N1pdm09 }\end{array}$ & $\begin{array}{l}\text { Anti } \\
\text { A/Victoria/2570/2019 } \\
\text { A(H1N1)pdm09 N156K }\end{array}$ \\
\hline $\begin{array}{l}\text { A/Victoria/2570/2019 } \\
\text { A(H1N1)pdm09 N156K }\end{array}$ & 80 & 320 & 1280 \\
\hline $\begin{array}{l}\text { A/Brandenburg/8/2020 } \\
\text { A(H1N1)pdm09 N156K }\end{array}$ & 160 & 320 & 1280 \\
\hline
\end{tabular}

Figure 1: Genetic characterization of viruses collected in season 2019-2020 $\left(\log _{2} \mathrm{HI}\right.$ titre reciprocal = HIU, hemagglutination inhibiting units $\left.\log _{2}\right)$. (A) A(H1N1)pdm09 viruses, (B) A/(H3N2) viruses); (C) B/Victoria viruses; (D) B/Yamagata viruses. Hemagglutination inhibition was performed using ferret antisera against A/Brisbane/02/2018 [ A(H1N1)pdm09 ], A/Kansas/14/2017 [ A/(H3N2) ], B/Colorado/6/2017 (B/Victoria) and B/Phuket/3073/2013 (B/Yamagata). Note that there was a good coverages of vaccine viruses in the influenza season 2019-2020 with the exception of N156K viruses.

Each rhombus represents a virus. Dark blue column, variation of the positive control (vaccine virus) $=1$ $\log _{2}$; light blue column, accepted tolerance in antigenic variation against vaccine viruses $=2 \log _{2}$; dark red column, viruses with N156K substitution in HA.

Figure 2: (A) Circulation of influenza viruses in the German influenza surveillance sentinel, influenza season 2019-2020 until now. (B) Percentage of N156K viruses among all $A(H 1 N 1) p d m 09$ viruses until the early end of influenza season due to lockdowns during the SARS-CoV-2 pandemic.

Figure 3: Phylogenetic analysis of HA (A) and NA (B) genes of A(H1N1)pdm09 viruses circulating in 2019-2020 (cw 42/2019-05/2020).

Virus genomes were analyzed by NGS and were phylogenetically evaluated with Mega7 (Neighbor-Joining method, bootstrap test with1000 replicates, Kimura 2-parameter method, partial deletion/site coverage cutoff 5\%). 164 influenza viruses were characterized, of which 59 (HA gene) and 58 (NA gene) viruses are shown in the phylogenetic analyzes. Almost all identical sequences are removed. Numbering of nucleotide positions (nt) starting from the first nucleotide of the gene (HA nt33 - nt1730, NA nt21 - nt1430). Substitutions in the deduced HA and NA amino acid sequences were identified by FluSurver database. The identified HA substitutions are displayed according to H1-numbering. H1-numbering starts after the signal peptide (-17). Mutations in the signal peptide are marked as sig pep. Polymorphisms are indicated as e.g. F242F/L. Virus specific substitutions are indicated for HA and NA sequences from Germany and additionally for the vaccine strain (2019-2020) A/Brisbane/02/2018. Furthermore, the identified substitutions are color-coded and assigned to the protein domains according to Igarashi $(2010,5)$ and Yang $(2010,12)$ : HA1 (black), HA2 (blue), antigenic site (red), receptor binding site (brown), both: antigenic site/receptor binding site (green). According to FluSurver the substitutions are highlighted that are significant for/associated with creation or removal of potential N-glycosylation site (pNLG) or antiviral drug resistance/reduced sensitivity.

The HA and NA sequences are marked as follows: viruses from 2019-2020-sentinel (black), 2019-2020non-sentinel (additionally NRZ), 2019-2020-N156K-variant (red), viruses from outbreaks in hospitals and old people's homes (outbreak), from vaccination breakthroughs after administration of trivalent (TIV) or quadrivalent (QIV) vaccine (2019-2020), viruses with reduced or no vaccine strain reactivity (HI40-640, noHI), viruses from patients with (respiratory) chronic pre-existing conditions (resp) chronicD), pneumonia, antiviral therapy, double infections with influenza and other viruses. The WHO reference sequences are marked in black / italics and the vaccine strains that were included in the 2019-2020 vaccine are red / italics. Intra-clade reassortants are framed.

The following abbreviations are used for the names of the virus isolates: BWB: Baden-Württemberg, 
BAY: Bavaria, BLN: Berlin, BBG: Brandenburg, BRE: Bremen, HAM: Hamburg, HES: Hessen, MVP: Mecklenburg-Vorpommern, NSA: Niedersachsen, NRW: North Rhine-Westphalia, RPF: RhinelandPalatinate, SAS: Saxony, SAT: Saxony-Anhalt, SAL: Saarland, SHO: Schleswig-Holstein, THR: Thuringia.

Figure 4: Structural models of $\mathrm{HA}$ and NA proteins of $\mathrm{A}(\mathrm{H} 1 \mathrm{~N} 1)$ pdm09 viruses detected in upper respiratory specimens. HA (A-C), NA (D-F). Viruses with HA-N156K substitution are analyzed by FluSurver database: A/MVP/2/2020/cluster 1 (A/C), A/MVP/12/2019/cluster 3 (B/E), A/NRW/142/2019/cluster 4 (C/F). Substitutions are relative to the closest reference A/Brisbane/02/2018 and the position of substitutions (H1-numbering) are highlighted if mutations are close to common drug, host receptor or antibody binding sites or if a glycosylation motif is lost or created through a mutation (black=no known effect/interestlevel 0 , green=subtype marker/interestlevel 0 , blue=site of interaction/ interestlevel 1 , orange $=$ drug binding/host-cell specificity/antigenic shifts/mild drug resistance/ interestlevel 2, magen$\mathrm{ta}=$ create/remove potential glycosylation site/ interestlevel 2, red=alter virulence/strong drug resistance/ interestlevel 3).

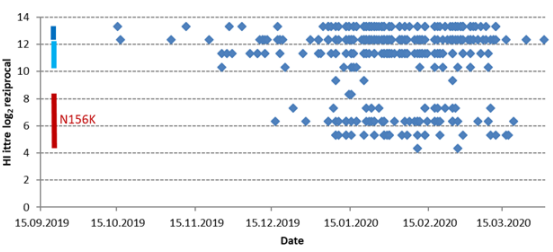

A

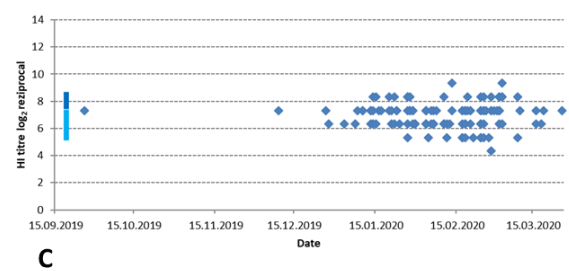

C

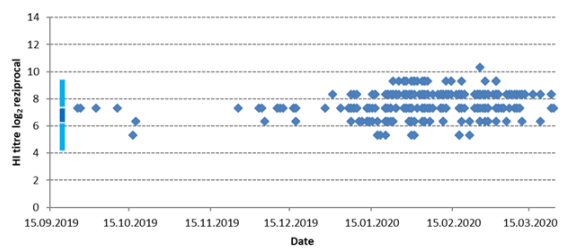

B

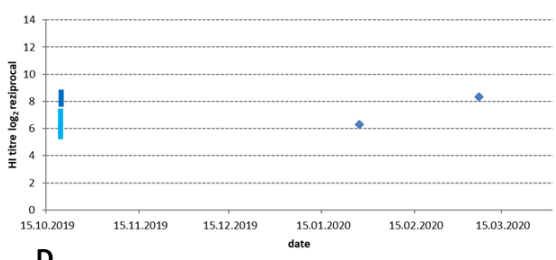

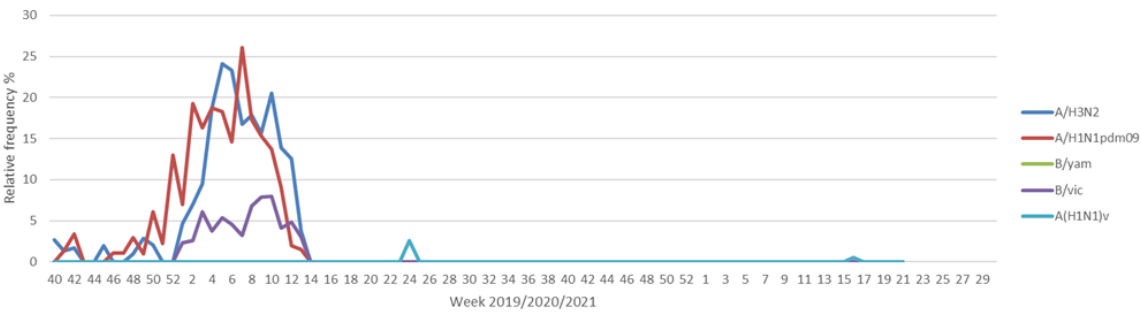

A

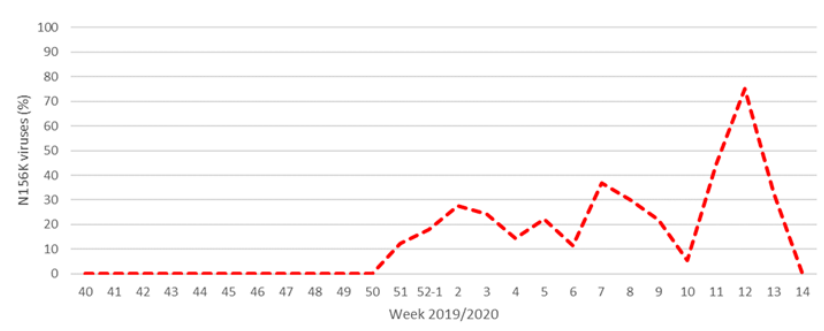

B 

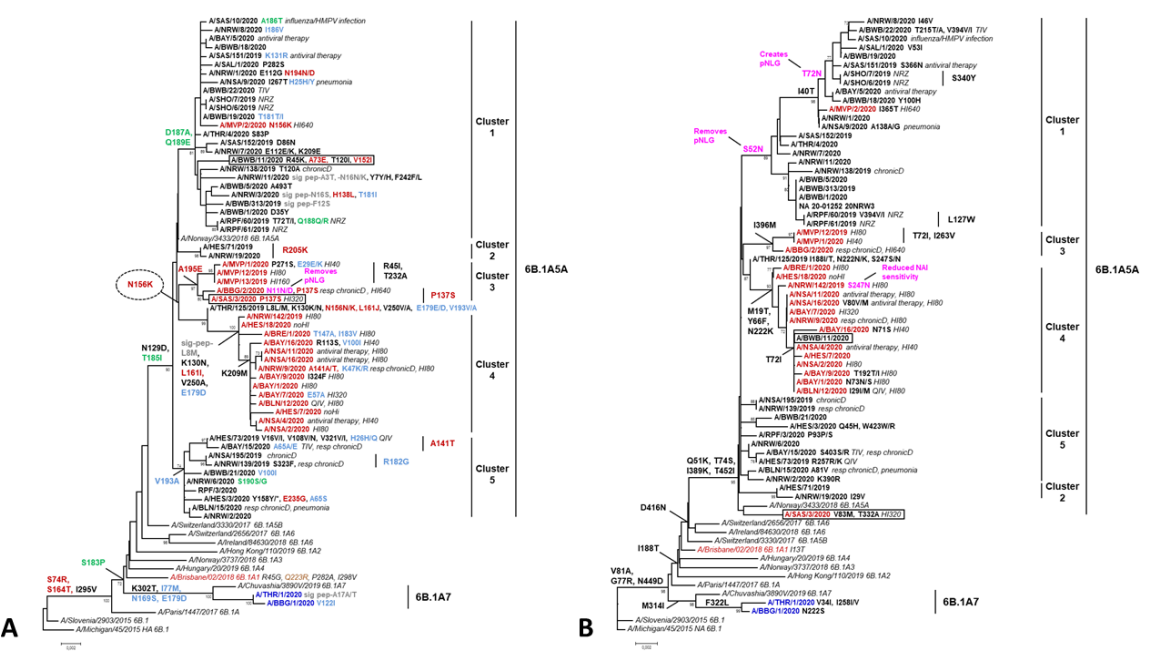

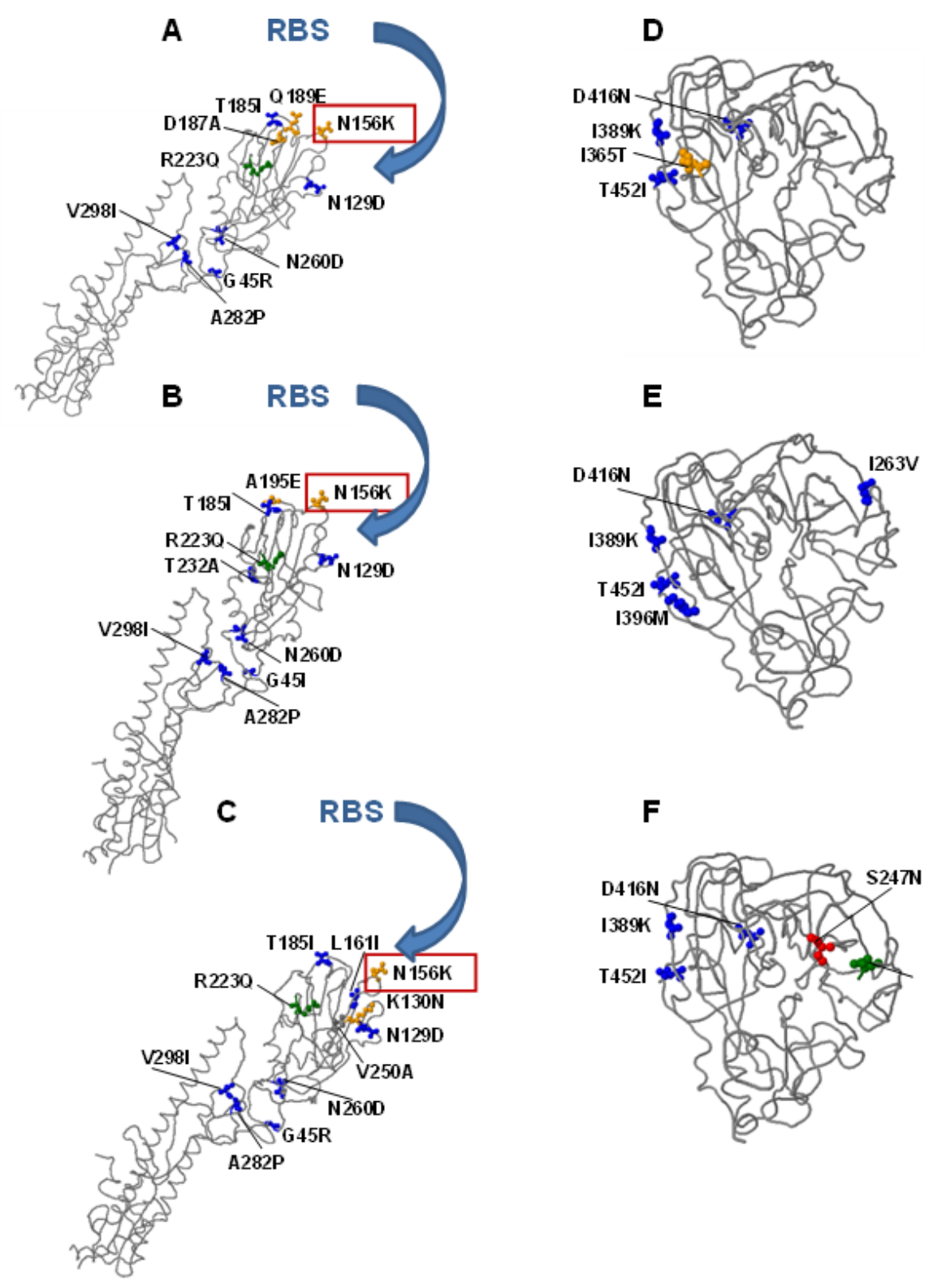